\title{
Performance of Parallel Decentralized Sensor Network with Decision Feedback
}

\author{
Sunitha Kopparthi and Balasubramaniam Natarajan \\ WiCom Group, Department of Electrical \& Computer Engineering \\ Kansas State University, Manhattan, Kansas 66506-5204 \\ Telephone: (785) 532-4674, Email: sunithak@ksu.edu
}

\begin{abstract}
In most previous studies, distributed sensor networks are typically assumed to be memory less. In this paper, we consider a distributed sensor network with feedback at both the sensor level as well as at the fusion center. Specifically, we analyze (1) a local feedback system $(L F S)$, where the most recent local decision is fed back to its corresponding local sensor; (2) a local and global feedback system 1 (LGFS1), where the most recent local decision is fed back to its corresponding local sensor and the most recent global decision is fed back to the fusion center, and (3) a local and global feedback system 2 (LGFS2), where the most recent global decision is fed back to all the local sensors and to the fusion center in addition to the local decision being fed back to its corresponding local sensor. For all the cases, we derive the decision rule and compare the global probability of error using simulations. We show that in an error-free channel, any form of feedback improves $G P E$ performance relative to no feedback system. However, feeding the global decision back to local sensors not only drains resources but also provides the worst performance among feedback schemes.
\end{abstract}

\section{INTRODUCTION}

Decentralized sensor network have attracted significant interest over past several years [1], [2] because of their reliability, survivability, and low communication bandwidth requirements. In a typical decentralized sensor network, local sensors make a decision about a phenomenon of interest based on an observation. The local sensors then transmit their sensors decisions to a fusion center. Global decision is made by the fusion center based on the local decisions made by the local sensors. A majority of prior efforts in decentralized detection in sensor networks have been confined to memoryless local sensors and fusion center. It is however possible to incorporate memory and use past decisions for the synthesis of new decisions.

Over the past decade, researchers ([3]-[9]), have shown that the global probability of error performance of a decentralized sensor network can be improved using decision feedback. In [4], [5], the fusion center calculates a global decision based on the decisions of local sensors, and this decision is fed back to the local sensors to adjust their detection thresholds. In an another approach [6], many local sensors reach a consensus through parley. In [7], [8], the authors proposed a concise feedback scheme where the previous global decision is fed back to the fusion center. Two distributed structures with decision feedback are considered in [9] where in the first distributed structure the fusion center decision is fed back to all the local sensors and in the second distributed structure all the local sensors are fully interconnected via decision feedback. While all these works have reiterated the importance of decision feedback at fusion center, they do not explicitly consider the effects of decision feedback at the local sensor level on the overall global probability of error $(G P E)$ performance.

Our main focus in this work is to study the effects of feedback, at both sensor level and fusion center, on the GPE performance of the sensor network. We consider three feedback schemes which offer a reasonable compromise between detection performance and complexity. The first proposed sensor network, local feedback system $(L F S)$, is a distributed decentralized sensor network, where the most recent local decision is fed back to its corresponding local detector. Global decision is obtained from threshold comparison of the weighted local decisions. In the second proposed sensor network, local \& global feedback system 1 (LGFS1), the most recent local decision is fed back to its corresponding local sensor and the most recent global decision is fed back to fusion center. The third sensor network, local \& global feedback system 2 (LGFS2), is an extension of sensor network LGFS1. Here, in addition to feedback at local sensors and fusion center, the most recent global decision is fed back to all the local sensors. LGFS2 scheme requires an additional communication path between the fusion center and the local sensors.

In this paper, we present a detailed description of these three systems and analyze their global probability of error (GPE) using theory and simulations. The performance of these systems is compared with decentralized sensor network without any feedback (no feedback system (NFS)) and decentralized sensor network presented in [8], where the previous global decision is fed back to the fusion center (global feedback system $(G F S)$ ). In case of an error-free channel, the $G P E$ performance of $L F S$ and $L G F S 1$ feedback systems are better than NFS. The GFS provides the best GPE performance relative to any other system illustrating that feedback at the fusion center is more effective than feedback at local sensors. LGFS2 does not provide any significant gain in GPE performance relative to NFS. Therefore, feeding global decision back to local sensors is not effective from a complexity standpoint as well as performance.

The three sensor network architectures with feedback ( $L F S$, $L G F S 1$ and $L G F S 2$ ) are described in Section II. GPE performance of these three sensor networks is investigated with an 
example in section III and is compared with the decentralized sensor networks NFS and GFS. Conclusions are discussed in section IV.

\section{SYSTEM MODEL}

A typical parallel distributed sensor network consists of a $N$ local sensors that observe a common phenomenon and make a binary decision. This binary decision could indicate the presence (hypothesis $H_{1}$ ) or absence (hypothesis $H_{0}$ ) of a target. For example, at each time instant, $t$, the $i$ th local detector obtains a new measurement, $Y_{i}$, and make a binary decision, $U_{i}^{(t)}$, where $U_{i}^{(t)}=-1$ indicates the absence of a target and hypothesis $H_{0}$ is accepted; $U_{i}^{(t)}=1$ indicates the presence of a target and hypothesis $H_{1}$ is accepted. We assume that (1) the sensor observations and the decisions are conditionally statistically independent in time as well as from sensor to sensor; (2) in the observation time window, the true hypothesis remains constant. It is also assumed that the local decisions are transmitted to fusion center over a noiseless communication channel. The fusion center makes a global decision, $U_{g}^{(t)}$, based on the local sensor decisions. Once again $U_{g}^{(t)}=-1$ represents the acceptance of hypothesis $H_{0}$ and $U_{g}^{(t)}=1$ indicates that hypothesis $H_{1}$ is accepted. The global decision is made by minimizing a Bayes risk [8] of the form

$$
\begin{aligned}
\beta\left(P_{f g}^{(t)}, P_{d g}^{(t)}\right) & =C_{00} P_{0}\left(1-P_{f g}^{(t)}\right)+C_{10} P_{0} P_{f g}^{(t)} \\
& +C_{01} P_{1}\left(1-P_{d g}^{(t)}\right)+C_{11} P_{1} P_{d g}^{(t)}
\end{aligned}
$$

where $C_{i j}$ is the cost of accepting $H_{i}$ when $H_{j}$ is true $(i, j=0,1), P_{j}$ is the apriori probability of hypothesis $H_{j}$ $(j=0,1)$, at time instant $t$, the global false alarm probability is $P_{f g}^{(t)}=P_{r}\left\{U_{g}^{(t)}=1 \mid H_{0}\right\}$ and the probability of detection is $P_{d g}^{(t)}=P_{r}\left\{U_{g}^{(t)}=1 \mid H_{1}\right\}$. Assuming a uniform cost function, i.e., $C_{00}=C_{11}=0$ and $C_{01}=C_{10}=1$, the Bayes risk is the global probability of error (GPE). With this general system model in place, we will discuss three different feedback architectures that can be implemented in a parallel decentralized sensor network.

1) Local Feedback System: In a local feedback system, the previous local sensor decision is used to obtain the present local decision as shown in Fig. 1. At time $t$, the final local decision, $U_{i f}^{(t)}$, is made based on the local decision, $U_{i}^{(t)}$, which is obtained from the current observation, $Y_{i}$, and the most recent local decision, $U_{i f}^{(t-1)}$. It is easy to show that the generalized rule for the local decision for the $L F S$ is given by

$$
U_{i f}^{(t)}=\operatorname{sgn}\left\{a_{i} U_{i}^{(t)}+a_{i f}^{(t-1)} U_{i f}^{(t-1)}-2 \log \tau-b_{i}-b_{i f}^{(t-1)}\right\}
$$

where

$$
\begin{gathered}
a_{i}=\mathbf{X}\left(P_{f}, P_{d}\right), b_{i}=\mathbf{Y}\left(P_{f}, P_{d}\right), \tau=P_{0} / P_{1}, \\
a_{i f}^{(t-1)}=\mathbf{X}\left(P_{f g_{-} l f}^{(t-1)}, P_{d g_{-} l f}^{(t-1)}\right), b_{i f}^{(t-1)}=\mathbf{Y}\left(P_{\text {fg llf }}^{(t-1)}, P_{d g_{-} l f}^{(t-1)}\right),
\end{gathered}
$$

$\operatorname{sgn}\{$.$\} is the algebraic sign function defined as$

$$
\operatorname{sgn}\{x\}=\left\{\begin{array}{ll}
1 & x \geq 0 \\
-1 & x<0
\end{array},\right.
$$

$P_{f}, P_{d}$ are the false alarm probability and probability of detection of the local detector. $P_{f g_{-} l f}, P_{d g_{-} l f}$ are the false alarm probability and the probability of detection of the local detector with feedback. $\tau$ is the decision threshold of the global detector. $\mathbf{X}$ and $\mathbf{Y}$ functions are defined as

$$
\mathbf{X}(a, b)=\log \left[\frac{b(1-a)}{a(1-b)}\right], \mathbf{Y}(a, b)=\log \left[\frac{a(1-a)}{b(1-b)}\right] .
$$

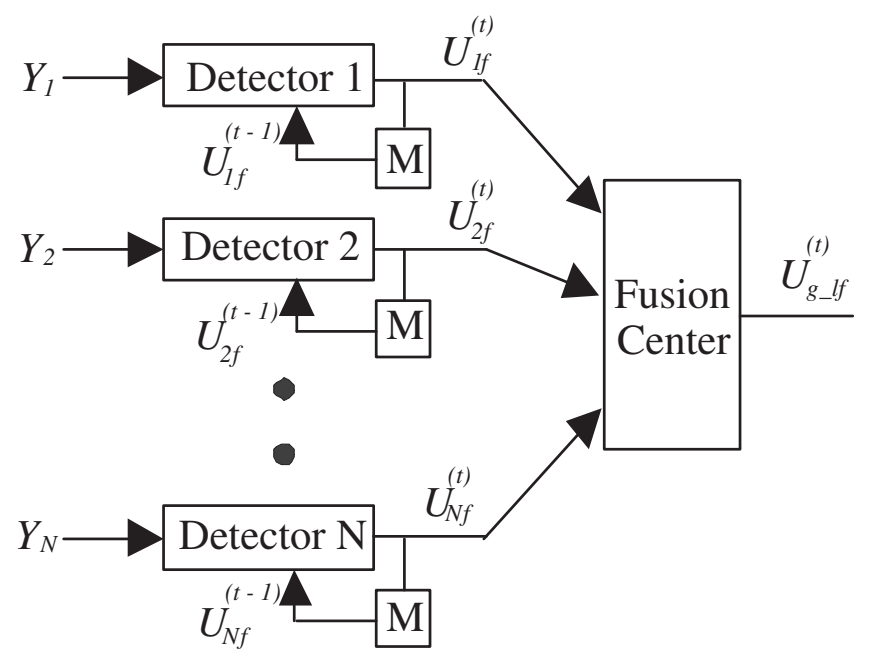

Fig. 1. Local Feedback System

The false alarm probability, $P_{f g \_l f}^{(t)}$, and the probability of detection, $P_{d g_{-} l f}^{(t)}$, of the local detector with feedback at time $t=1,2, \ldots$ can be expressed in terms of the $P_{f g_{-l f}}^{(t-1)}, P_{d g-l f}^{(t-1)}$, $P_{f}, P_{d}$ and $n$ (where $n$ is an integer) through the following iterative scheme [8]:

$$
\begin{aligned}
P_{f g-l f}^{(t)} & =\mathbf{F}\left(P_{f g-l f}^{(t-1)}, P_{d g_{-l f}}^{(t-1)}, P_{f}, 1\right), \\
P_{d g_{-} l f}^{(t)} & =\mathbf{D}\left(P_{\text {fg-lf }}^{(t-1)}, P_{d g_{-} l f}^{(t-1)}, P_{d}, 1\right),
\end{aligned}
$$

where

$$
\begin{gathered}
\mathbf{F}(x, y, A, n)=\sum_{i=0}^{n}\left(\begin{array}{c}
n \\
i
\end{array}\right) A^{i}(1-A)^{n-i}[(1-x) \\
\left.U_{-1}\left(\gamma_{i}(x, y, A, B, n)\right)+x U_{-1}\left(\delta_{i}(x, y, A, B, n)\right)\right], \\
\mathbf{D}(x, y, B, n)=\sum_{i=0}^{n}\left(\begin{array}{c}
n \\
i
\end{array}\right) B^{i}(1-B)^{n-i}[(1-y) \\
\begin{array}{r}
\left.U_{-1}\left(\gamma_{i}(x, y, A, B, n)\right)+y U_{-1}\left(\delta_{i}(x, y, A, B, n)\right)\right], \\
\gamma_{i}(x, y, A, B, n)=B^{i}(1-B)^{n-i}(1-y) \\
-\quad \tau A^{i}(1-A)^{n-i}(1-x), \\
\delta_{i}(x, y, A, B, n)=B^{i}(1-B)^{n-i} y-\tau A^{i}(1-A)^{n-i} x .
\end{array}
\end{gathered}
$$


Here, $U_{-1}($.$) is a unit step function defined by$

$$
U_{-1}(x)=\left\{\begin{array}{ll}
1 & x \geq 0 \\
0 & x<0
\end{array} .\right.
$$

Intially, we assume that $P_{f g_{-} l f}^{(0)}$ and $P_{d g_{-} l f}^{(0)}$ are equal to 0.5 . These equations are based on the definitions

$$
\begin{aligned}
P_{f g l f}^{(t)} & =P_{r}\left\{U_{i f}^{(t)}=1 \mid H_{0}\right\} \\
& =\mathbf{E}\left[P_{r}\left\{U_{i f}^{(t)}=1 \mid H_{0}, U_{i}^{(t)}, U_{i f}^{(t-1)}\right\}\right], \\
P_{d g l f}^{(t)} & =\operatorname{Pr}\left\{U_{i f}^{(t)}=1 \mid H_{1}\right\} \\
& =\mathbf{E}\left[P_{r}\left\{U_{i f}^{(t)}=1 \mid H_{1}, U_{i}^{(t)}, U_{i f}^{(t-1)}\right\}\right],
\end{aligned}
$$

where $\mathbf{E}$ [.] is an expectation function evaluated over all local decisions. The fusion rule for global decision is obtained by

$$
U_{g_{-} l f}^{(t)}=\operatorname{sgn}\left\{\sum_{i=1}^{N} a_{g}^{(t)} U_{i f}^{(t)}-2 \log \tau-b_{g}^{(t)}\right\}
$$

where

$$
a_{g}^{(t)}=\mathbf{X}\left(P_{f g_{-} l f}^{(t)}, P_{d g_{-} l f}^{(t)}\right), b_{g}^{(t)}=N \cdot \mathbf{Y}\left(P_{f g_{-} l f}^{(t)}, P_{d g_{-} l f}^{(t)}\right),
$$

$\mathbf{X}$ and $\mathbf{Y}$ are defined in Eqn. (3).

Using the global fusion rule Eqn. (5), the global probability of error is found by [8]

$$
G P E=\beta\left(P_{f g}^{(t)}, P_{d g}^{(t)}\right)=P_{0} P_{f g}^{(t)}+P_{1}\left(1-P_{d g}^{(t)}\right)
$$

where $P_{f g}^{(t)}$ and $P_{d g}^{(t)}$ are the global false alarm probability and global probability of detection given by

$$
\begin{aligned}
& \quad P_{f g}^{(t)}=\mathbf{G}\left(P_{f g_{-} l f}^{(t)}, N, z\right), \quad P_{d g}^{(t)}=\mathbf{G}\left(P_{d g_{-} l f}^{(t)}, N, z\right), \\
& z= \\
& \mathbf{I}\left(P_{f g_{-} l f}^{(t)}, P_{d g_{-} l f}^{(t)}, N, \tau\right)
\end{aligned}
$$

with

$$
\mathbf{G}(u, v, w)=\sum_{i=w}^{v}\left(\begin{array}{c}
v \\
i
\end{array}\right) u^{i}(1-u)^{v-i}
$$

$\mathbf{I}(p f, p d, c, d)=\operatorname{int}\left\{\frac{\ln (d)+c \cdot \ln [(1-p f) /(1-p d)]}{\ln (p d / p f)+\ln [(1-p f) /(1-p d)]}\right\}$

where $i n t\{k\}$ is the smallest integer larger than or equal to $k: k \leq \operatorname{int}\{k\}<k+1$. It is important to note that the analysis presented above can be extended to the case where there is feedback of more than one past local decision. However, since the objective of this work is to compare the effectiveness of various feedback architectures, we use the one-stage feedback as a standard for all methods considered.

In the following sub sections, we present two additional feedback structures referred to as LGFS1 and LGFS2. In both these implementations, we propose a feedback of decisions both at the local sensor level and at the fusion center. For these cases, it is not straight forward to derive the optimal global decision rule because the global decision is no longer based on an independent set of decisions. The global decision is based on local decisions which in turn depends on the previous local decision (thanks to the feedback mechanism) introducing dependance among the components contributing to the global decision. Therefore, the decision rules for LGFS1 \& LGFS2 presented in the next two sub-sections are sub-optimal with respect to GPE performance. The simulation results provided in section III provide the realistic GPE performance based on these decision rules.

2) Local and Global Feedback System 1: In local and global feedback system 1, shown in Fig. 2, the local decision is made based on its current observation and its most recent local decision. The global decision is made based on all final local decisions and the most recent global decision. At time $t$, the final local decision, $U_{i l g f 1}^{(t)}$, is made based on the local decision, $U_{i}^{(t)}$, which is obtained from the current observation, $Y_{i}$, and the previous local decision, $U_{i l g f l}^{(t-1)}$. The generalized

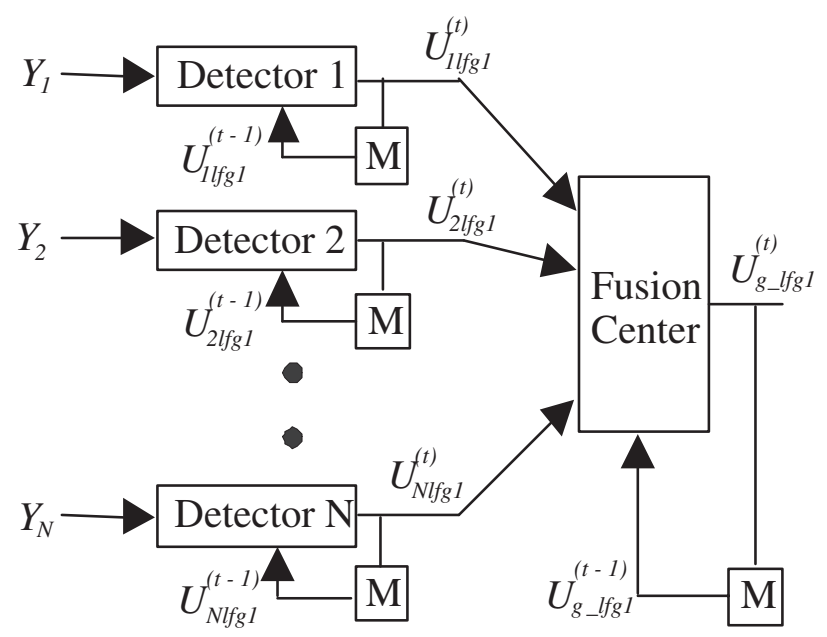

Fig. 2. Global and Local Feedback System 1

rule of the final local decision for the LGFS1 is expressed as

$$
\begin{array}{r}
U_{i l g f 1}^{(t)}=\operatorname{sgn}\left\{a_{i} U_{i}^{(t)}+a_{i l g f 1}^{(t-1)} U_{i l g f 1}^{(t-1)}-2 \log \tau\right. \\
\left.-b_{i}-b_{i l g f 1}^{(t-1)}\right\}
\end{array}
$$

where

$$
a_{i l g f 1}^{(t-1)}=\mathbf{X}\left(P_{f_{-} l g f 1}^{(t-1)}, P_{d_{-} l g f 1}^{(t-1)}\right), b_{i l g f 1}^{(t-1)}=\mathbf{Y}\left(P_{f_{-} l g f 1}^{(t-1)}, P_{d_{-} l g f 1}^{(t-1)}\right) .
$$

$P_{f_{-} l g f 1}$ is the false alarm probability and $P_{d_{-} l g f 1}$ is the probability of detection of the local sensor with feedback and could be found by using the following formula.

$$
\begin{aligned}
P_{f \_l g f 1}^{(t)} & =\mathbf{F}\left(P_{f_{-l g f 1}(t-1)}^{(t)}, P_{d \_l g f 1}^{(t-1)}, P_{f}, 1\right), \\
P_{d \_l g f 1}^{(t)} & =\mathbf{D}\left(P_{f_{-} l g f 1}^{(t-1)}, P_{d \_l g f 1}^{(t-1)}, P_{d}, 1\right),
\end{aligned}
$$

where $\mathbf{X}, \mathbf{Y}$ are defined in Eqn. (3) and F, $\mathbf{D}$ are defined in Eqn. (4). The fusion rule for global decision is obtained by

$$
\begin{array}{r}
U_{g_{-} l g f 1}^{(t)}=\operatorname{sgn}\left\{\sum_{i=1}^{N} a_{f g 1}^{(t)} U_{i l g f 1}^{(t)}+a_{g_{-} l g f 1}^{(t-1)} U_{g_{-} l g f 1}^{(t-1)}\right. \\
\left.-2 \log \tau-b_{f g 1}^{(t)}-b_{g_{-} l g f 1}^{(t-1)}\right\}
\end{array}
$$


where

$$
\begin{aligned}
& a_{f g 1}^{(t)}=\mathbf{X}\left(P_{f_{l} l g f 1}^{(t)}, P_{d_{-} l g f 1}^{(t)}\right), b_{f g 1}^{(t)}=N \cdot \mathbf{Y}\left(P_{f_{-l g f 1}^{(t)}}^{(t)}, P_{d_{-l} l g f 1}^{(t)}\right), \\
& a_{g_{\_} l g f 1}^{(t-1)}=\mathbf{X}\left(P_{f g_{-} l g f 1}^{(t-1)}, P_{d g_{-} \lg f 1}^{(t-1)}\right), \\
& b_{g_{\iota} l g f 1}^{(t-1)}=\mathbf{Y}\left(P_{f g_{-} l g f 1}^{(t-1)}, P_{d g_{\_} l g f 1}^{(t-1)}\right), \\
& P_{f g l g f 1}^{(t)}=\mathbf{F}\left(P_{f g_{-l g f 1}}^{(t-1)}, P_{d g \_l g f 1}^{(t-1)}, P_{f_{-} l g f 1}, N\right), \\
& P_{d d_{-} l g f 1}^{(t)}=\mathbf{D}\left(P_{f g_{-} l g f 1}^{(t-1)}, P_{d g_{-} l g f 1}^{(t-1)}, P_{d_{-} l g f 1}, N\right) .
\end{aligned}
$$

$P_{f g_{-} l g f 1}^{(t)}$ and $P_{d g_{l} l g f 1}^{(t)}$ are the global false alarm probability and global probability of detection respectively. Intially it is assumed that $P_{f g_{-} l g f 1}^{(0)}=P_{d g_{-} l g f 1}^{(0)}=0.5$. Based on the decision rule of Eqn. (8) the GPE for LGFS1 can be obtained and are provided in section III.

3) Local and Global Feedback System 2: In local and global feedback system 2, shown in Fig. 3, the global decision is made based on all final local decisions and the most recent global decision. At time $t$, the final local decision, $U_{i l g f 2}^{(t)}$, is made based on the local decision, $U_{i}^{(t)}$, which is obtained from the current observation, $Y_{i}$, and the previous local decision, $U_{i l g f 2}^{(t-1)}$, and the most recent global decision $U_{g l g f 2}^{(t-1)}$. The generalized rule of the final local decision for the LGFS2 is expressed as

$$
\begin{aligned}
U_{i l g f 2}^{(t)}=\operatorname{sgn}\left\{a_{i} U_{i}^{(t)}\right. & +a_{i l g f 2}^{(t-1)} U_{i l g f 2}^{(t-1)}+a_{\text {gllgf2 } 2}^{(t-1)} U_{g_{l} l g f 2}^{(t-1)} \\
& \left.-2 \log \tau-b_{i}-b_{i l g f 2}^{(t-1)}-b_{g_{-} \operatorname{lgf} 2}^{(t-1)}\right\}
\end{aligned}
$$

where

$$
\begin{aligned}
& a_{i l g f 2}^{(t-1)}=\mathbf{X}\left(P_{f_{-} l g f 2}^{(t-1)}, P_{d_{-} \operatorname{lgf} 2}^{(t-1)}\right), b_{i l g f 2}^{(t-1)}=\mathbf{Y}\left(P_{f_{-} \lg f 2}^{(t-1)}, P_{d_{-} l g f 2}^{(t-1)}\right),
\end{aligned}
$$

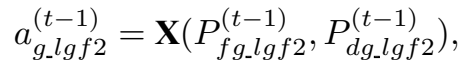

$$
\begin{aligned}
& b_{g_{\iota} l g f 2}^{(t-1)}=\mathbf{Y}\left(P_{f g_{-} l g f 2}^{(t-1)}, P_{d g_{-} l g f 2}^{(t-1)}\right) .
\end{aligned}
$$

$P_{f_{-} l g f 2}$ is the false alarm probability and $P_{d_{-} l g f 2}$ is the probability of detection of the local sensor with feedback and could be found by using the following formula.

$$
\begin{aligned}
P_{f_{-l g f 2}^{(t)}}^{(t)} & =\mathbf{F}\left(P_{f_{-l g f 2}^{(t-1)}}^{(t-1)}, P_{d \_l g f 2}^{(t-1)}, P_{f}, 2\right), \\
P_{d \_l g f 2}^{(t)} & =\mathbf{D}\left(P_{f_{-} l g f 2}^{(t-1)}, P_{d_{-l g f 2}}^{(t-1)}, P_{d}, 2\right),
\end{aligned}
$$

$\mathbf{X}, \mathbf{Y}$ are defined in Eqn. (3) and $\mathbf{F}, \mathbf{D}$ are defined in Eqn. (4). $P_{f g_{\_} l g f 2}$ and $P_{d g_{-} l g f 2}$ are the global probability of false alarm and the global probability of detection of the entire system can be expressed as

$$
\begin{gathered}
P_{f g l l g f 2}^{(t)}=\mathbf{F}\left(P_{f g \_l g f 2}^{(t-1)}, P_{d g \_l g f 2}^{(t-1)}, P_{f_{-} l g f 2}, N\right) \\
P_{d g_{l} l g f 2}^{(t)}=\mathbf{D}\left(P_{f g_{-} l g f 2}^{(t-1)}, P_{d g_{-} l g f 2}^{(t-1)}, P_{d_{-} l g f 2}, N\right) .
\end{gathered}
$$

Intially it is assumed that $P_{f_{-l} g f 2}^{(0)}, P_{d \_l g f 2}^{(0)}, P_{f g_{-} l g f 2}^{(0)}$ and

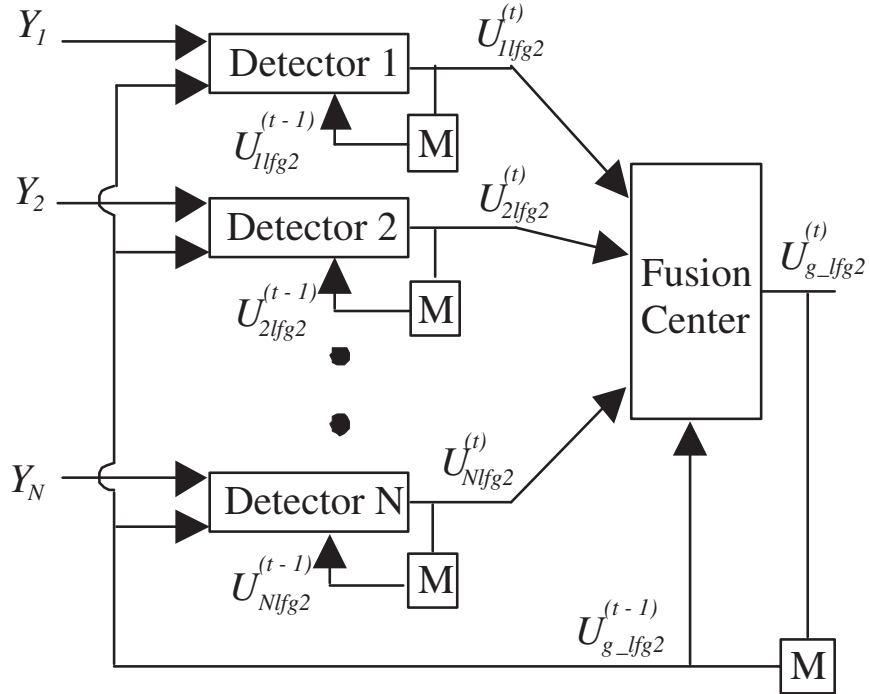

Fig. 3. Global and Local Feedback System 2

$P_{d g \_l g f 2}^{(0)}$ are equal to 0.5 . The fusion rule for global decision is obtained by

$$
\begin{aligned}
& U_{g_{\iota} l g f 2}^{(t)}=\operatorname{sgn}\left\{\sum_{i=1}^{N} a_{f g 2}^{(t)} U_{i l g f 2}^{(t)}+a_{g_{\iota} l g f 2}^{(t-1)} U_{g_{\iota} l g f 2}^{(t-1)}\right. \\
& \left.-2 \log \tau-b_{f g 2}-b_{g_{-} l g f 2}^{(t-1)}\right\}
\end{aligned}
$$

where

$$
a_{f g 2}^{(t)}=\mathbf{X}\left(P_{f_{-} l g f 2}^{(t)}, P_{d_{-} l g f 2}^{(t)}\right), b_{f g 2}^{(t)}=N \cdot \mathbf{Y}\left(P_{f_{-} l g f 2}^{(t)}, P_{d_{-} l g f 2}^{(t)}\right) .
$$

In $L G F S 2$, feeding back the global decision to local sensors increases the complexity of the system as a dedicated communication path from the fusion center to the local sensors is required. Based on the decision rule of Eqn. (10) the GPE for $L G F S 2$ can be obtained and are provided in the next section.

\section{Performance Results}

In this section, the performance of $L F S, L G F S 1$ and $L G F S 2$ are investigated with an example and are compared with NFS and GFS cases. Consider a 4 sensor system where the receiver operating characteristic of the local detector is defined as:

$$
P_{d}=P_{f}^{1 /(1+r)}
$$

where $r$ is the average signal-to-noise ratio. In many detection problems, this kind of receiver operating characteristics occur in classifying Gaussian signals with equal means and different variances [10]. Let the variances under hypothesis $H_{0}$ and $H_{1}$ be $\sigma_{0}^{2}$ and $\sigma_{1}^{2}$ respectively. The signal-to-noise ratio is defined as $r=\left(\sigma_{1}^{2}-\sigma_{0}^{2}\right) / \sigma_{0}^{2}$. For NFS, the GPE performance is given by $[8]$

$$
\begin{array}{r}
G P E=\beta\left(P_{f g}^{(M)}, P_{d g}^{(M)}\right), P_{f g}^{(M)}=\mathbf{G}\left(P_{f}, N, z 1\right), \\
P_{d g}^{(M)}=\mathbf{G}\left(P_{d}, N, z 1\right), z 1=\mathbf{I}\left(P_{f}, P_{d}, N, \tau\right)
\end{array}
$$

where $\mathbf{G}$, and $\mathbf{I}$ are defined in Eqn. (6). The GPE performance of the GFS is given by

$$
G P E=P_{0} P_{f g f}^{(t)}+P_{1}\left(1-P_{d g f}^{(t)}\right)
$$


where

$$
\begin{aligned}
& P_{f g f}^{(t)}=\mathbf{F}\left(P_{f g f}^{(t-1)}, P_{d g f}^{(t-1)}, P_{f}, N\right) \\
& P_{d g f}^{(t)}=\mathbf{D}\left(P_{f g f}^{(t-1)}, P_{d g f}^{(t-1)}, P_{d}, N\right)
\end{aligned}
$$

and $\mathbf{F}, \mathbf{D}$ are defined in (4). For $N=4, P_{f}=0.2$ and $\tau=0.5$, the global probability of error with respect to the signal-tonoise ratio of the local detector is plotted. The performance of the GPE vs. signal-to-noise ratio for $L F S, N F S$ and $G F S$ in case of an error-free communication channel is shown in Fig. 4. The discontinuous nature of the GPE performance of $L F S$ and $G F S$ feedback systems is a result of the discontinuities in the functions $\mathbf{F}$ and $\mathbf{D}$ in Eqn. (4). From Fig. 4, it can be observed that the GPE performance of global feedback system is significantly better relative to local feedback and no feedback systems. The simulated performance of $L F S$ is

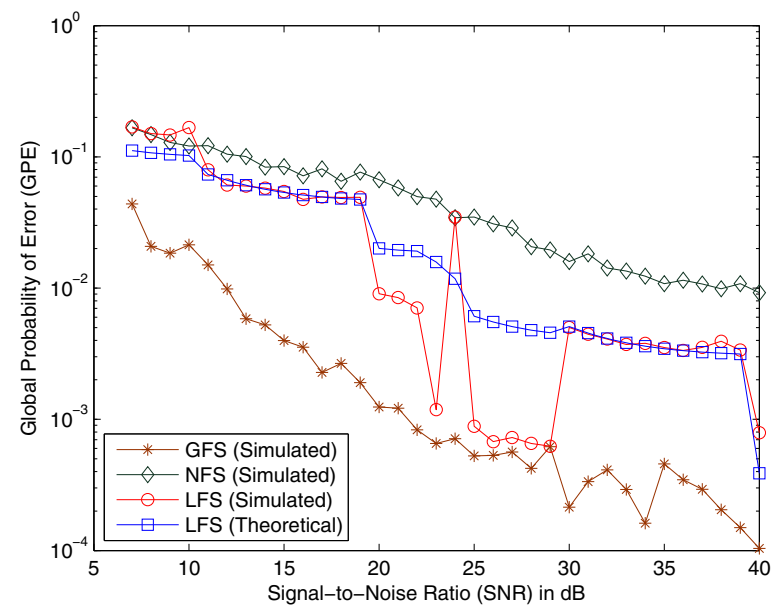

Fig. 4. GPE, vs. Signal-to-noise ratio, (r), for $N F S, L F S$ and $G F S$ for error-free channel with parameters $N=4, P_{f}=0.2, \tau=0.5$ and $P_{d}^{1 /(1+r)}$

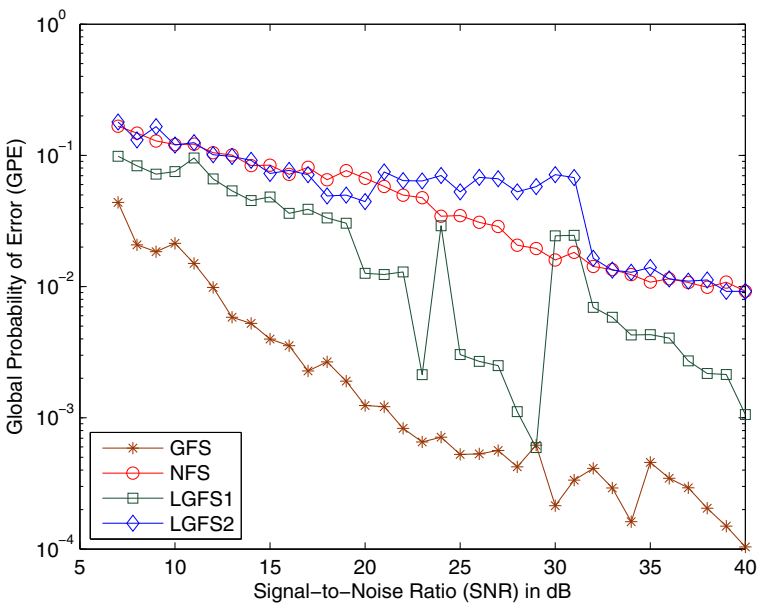

Fig. 5. GPE, vs. Signal-to-noise ratio, (r), for $N F S, G F S, L G F S 1$ and $L G F S 2$ for error-free channel with parameters $N=4, P_{f}=0.2, \tau=0.5$ and $P_{d}^{1 /(1+r)}$ comparable with theoretical performance. Fig. 4 shows that any form of feedback either at the local sensor level or at the fusion center improves the GPE performance.

The GPE performance vs. signal-to-noise ratio for $G F S$, $L G F S 1$ and LGFS2 for an error-free channel is shown in Fig. 5. From Figs. 4 and 5, it can be observed that the GFS system provides the best $G P E$ performance relative to all other feedback systems. The GPE performance of $L G F S 1$ is comparable to that of $L F S$. However, the $L G F S 2$ provides no performance gain relative to $N F S$ inspite of the added complexity. Hence this method is not efficient when compared to any other feedback methods.

\section{CONCLUSIONS}

In this paper, three feedback architectures for a parallel distributed sensor network are considered. In the first feedback system $(L F S)$, the local sensor decisions are made based on the local sensor observation and its most recent local decision. In the second feedback system (LGFS1), in addition to the local feedback the most recent global decision is fed to the fusion center. In the third feedback system ( $L G F S 2)$, in addition to the local and global feedback the most recent global decision is fed back to all the local sensors. Global probability of error (GPE) performance of these three feedback systems are evaluated. The simulated GPE performance of LFS, LGFS1 and LGFS2 are compared with $G F S$ and $N F S$ for error-free communication channel. It is observed that $G F S$ provides the best performance relative to any other feedback system. $L G F S 2$ does not provide any significant gain in GPE performance relative to NFS. Therefore, feeding global decision back to local sensors is not effective from a complexity standpoint as well as performance.

\section{REFERENCES}

[1] R. R. Tenney and N. R. Sandell, "Detection with Distributed Sensors," IEEE Trans. Aerosp. Electron. Syst., vol. AES-17, no. 4, pp. 501-509, July 1981.

[2] Z. Chair and P. K. Varshney, "Optimal Data Fusion in Multiple Sensor Detection Systems," IEEE Trans. Aerosp. Electron. Syst., vol. AES22, no. 1, pp. 98-101, Jan. 1986.

[3] R. Viswanathan and P. K. Varshney, "Distributed Detection with Multiple Sensors: part I - Fundamentals," Proceedings of the IEEE, vol. 85, no. 1, pp. 54-63, Jan. 1997.

[4] R. Srinivasan, "Distributed Detection with Decision Feedback," IEE Proc. F, Commun, Radar \& Signal Process., vol. 137, no. 6, pp. 427 - 432, Dec. 1990

[5] S. Alhakeem and P. K. Varshney, "Decentralized Bayesian Detection with Feedback," IEEE Trans. Syst., Man, Cybern. A, vol. 26, no. 4, pp. 503 - 513, July 1996.

[6] P. F. Swaszek and P. Willett, "Parley as an approach to distributed detection," IEEE Trans. Aerosp. Electron. Syst., vol. 31, no. 1, pp. 447 - 457, Jan. 1995

[7] J. Guan, Y. He and Y. Peng, "Distributed Detection with Feedback and Optimal Fusion," The Record of the IEEE 2000 International Radar Conference, pp. 151 - 154, May 2000.

[8] M. Kam, C. Rorres, W. Chang and X. Zhu, "Performance and Geometric Interpretation for Decision Fusion with Memory," IEEE Trans. Syst., Man, Cybern. A, vol. 29, no. 1, pp. 52 - 62, Jan. 1999.

[9] D. A. Pados, K. W. Halford, D. Kazakos and P. Papantoni-Kazakos, "Distributed Binary Hypothesis Testing with Feedback," IEEE Trans. Syst., Man, Cybern., vol. 25, no. 1, pp. 21 - 42, Jan. 1995.

[10] H. L. Van Trees, "Detection, Estimation, and Modulation Theory," New York: Wiley, 1969. 